\title{
Disease and health management in Asian aquaculture
}

\begin{abstract}
Asia contributes more than $90 \%$ to the world's aquaculture production. Like other farming systems, aquaculture is plagued with disease problems resulting from its intensification and commercialization. This paper describes the various factors, providing specific examples, which have contributed to the current disease problems faced by what is now the fastest growing food-producing sector globally. These include increased globalization of trade and markets; the intensification of fish-farming practices through the movement of broodstock, postlarvae, fry and fingerlings; the introduction of new species for aquaculture development; the expansion of the ornamental fish trade; the enhancement of marine and coastal areas through the stocking of aquatic animals raised in hatcheries; the unanticipated interactions between cultured and wild populations of aquatic animals; poor or lack of effective biosecurity measures; slow awareness on emerging diseases; the misunderstanding and misuse of specific pathogen free (SPF) stocks; climate change; other human-mediated movements of aquaculture commodities. Data on the socio-economic impacts of aquatic animal diseases are also presented, including estimates of losses in production, direct and indirect income and employment, market access or share of investment, and consumer confidence; food availability; industry failures. Examples of costs of investment in aquatic animal health-related activities, including national strategies, research, surveillance, control and other health management programmes are also provided. Finally, the strategies currently being implemented in the Asian region to deal with trans boundary diseases affecting the aquaculture sector are highlighted. These include compliance with international codes, and development and implementation of regional guidelines and national aquatic animal health strategies; new diagnostic and therapeutic techniques and new information technology; new biosecurity measures including risk analysis, epidemiology, surveillance, reporting and planning for emergency response to epizootics; targeted research; institutional strengthening and manpower development (education, training and extension research and diagnostic services).
\end{abstract}

Keyword: Aquaculture; Parasite disease; Production loss; Disease management 